\title{
VERS UNE REMISE EN GÉOMÉTRIE AUTOMATIQUE DES PRISES DE VUES AÉRIENNES HISTORIQUES PHOTOGRAMMÉTRIQUES
}

\author{
Arnaud Le Bris, Sébastien Giordano, Clément Mallet \\ Univ. Paris-Est, LASTIG MATIS, IGN, ENSG, 73 Avenue de Paris, F-94160 Saint-Mandé, France
}

\begin{abstract}
Résumé
Les prises de vues aériennes photogrammétriques historiques (antérieures aux premières images satellites) sont aujourd'hui disponibles numérisées dans de nombreux pays, le plus souvent de manièr etotalement libre. Elles sont un outil unique et assez peu exploité pour le suivi des changements 3D de l'occupation des sols au cours du siècle passé. Ces études restent toutefois limitées en termes de surface et de nombre de dates concernées, du fait de l'absence de méthode automatique pour géoréférencer finement les images anciennes. Le principal verrou réside dans l'identification de points d'appui en nombre et en densité suffisants. Dans ce but, cet article propose une approche photogrammétrique en deux étapes. La mise en place absolue des images est d'abord calculée automatiquement de manière approchée à partir des métadonnées basiques associées aux images d'archives. Elles est suivie d'une détection automatique de points d'appui utilisés ensuite pour l'amélioration de ce géoréférencement. Ortho-images et Modèles Numériques de Surface récents sont utilisés comme référence pour toutes les dates. La détection automatique de points d'appui se fait par comparaison de cette ortho-image de référence avec celle obtenue à partir du géoréférencement approché des clichés anciens. Une aérotriangulation de la prise de vues peut alors être calculée à partir de ces points. Nos expérimentations sur deux zones d'étude (urbain et forêt) montrent la validité de notre approche et la bonne qualité des résultats. Elles permettent d'ores et déjà dans de nombreux cas d'applications thématiques l'exploitation des images aériennes historiques en trois dimensions.
\end{abstract}

Mots clés : Images aériennes, archives, 3D, orientation, points d'appui, Modèle Numérique de Surface, ortho-image.

\begin{abstract}
Archival photogrammetric aerial campaigns are a unique and relatively unexplored means to chronicle 3D land-cover changes over the past 100 years. They have been digitized in many countries so far and are even available for free. However, existing studies are rather limited in terms of area and number of dates since no fully automatic method has been proposed yet to finely georeference archival images, the major challenge being the identification of a sufficient amount of ground references : cartographic coordinates and their position in the archival images. This paper adresses this issue and proposes a photogrammetric 2-step approach. First, a coarse absolute image orientation is computed out of basic metadata provided with the archives. Then, an automatic extraction of ground control points is performed. These points are used in order to refine orientation. Such a step only relies on a recent orthoimage+Digital Surface Model, used as reference for all epochs. The coarse orthoimage derived out of coarsely oriented archival images is compared with such a reference, and enables the identification of dense ground references. At the end, a new bundle adjustment using these ground control points is calculated. Our experiments on two distinct areas (one urban and oen forested) show the validity of our approach and the satisfactory quality of the results. They already allow the exploitation of the images for various thematical use cases.
\end{abstract}

Keywords : Aerial images, archives, 3D, orientation, Ground Control Points, Digital Surface Model, orthoimage.

\section{Introduction}

Les prises de vues aériennes photogrammétriques disponibles aujourd'hui dans les archives ont été acquises initialement par les agences cartographiques, cadastrales et militaires au cours de campagnes systématiques à des fins de production de cartes et de données topographiques. II s'agissait alors d'acquisitions stéréoscopiques afin de permettre une restitution tridimensionnelle des objets. Ces campagnes de prises de vues ont été réalisées de manière systématique et régulière dans de nombreux pays au cours du siècle dernier et ce dès les années 1910-1930. Récemment, dans différents pays, les clichés argentiques présents dans les archives des instituts cartographiques ont fait l'objet de numérisations. Leur mise à la disposition, la plupart du temps gratuite, auprès d'utilisateurs potentiels a été facilitée par la mise en place d'infrastructures et de services en ligne assurant leur visualisation et leur diffusion ainsi que celle de métadonnées de base les concernant. A titre d'exemple, en France, ces données sont diffusées via la plateforme "Remonter le temps" ( https ://remonterletemps.ign.fr/ ) de l'Institut National de l'Information Géographique et Forestière (IGN). Cela représente aujourd'hui plus de 3 millions de clichés acquis depuis le début du XXème siècle (Figure 1). 

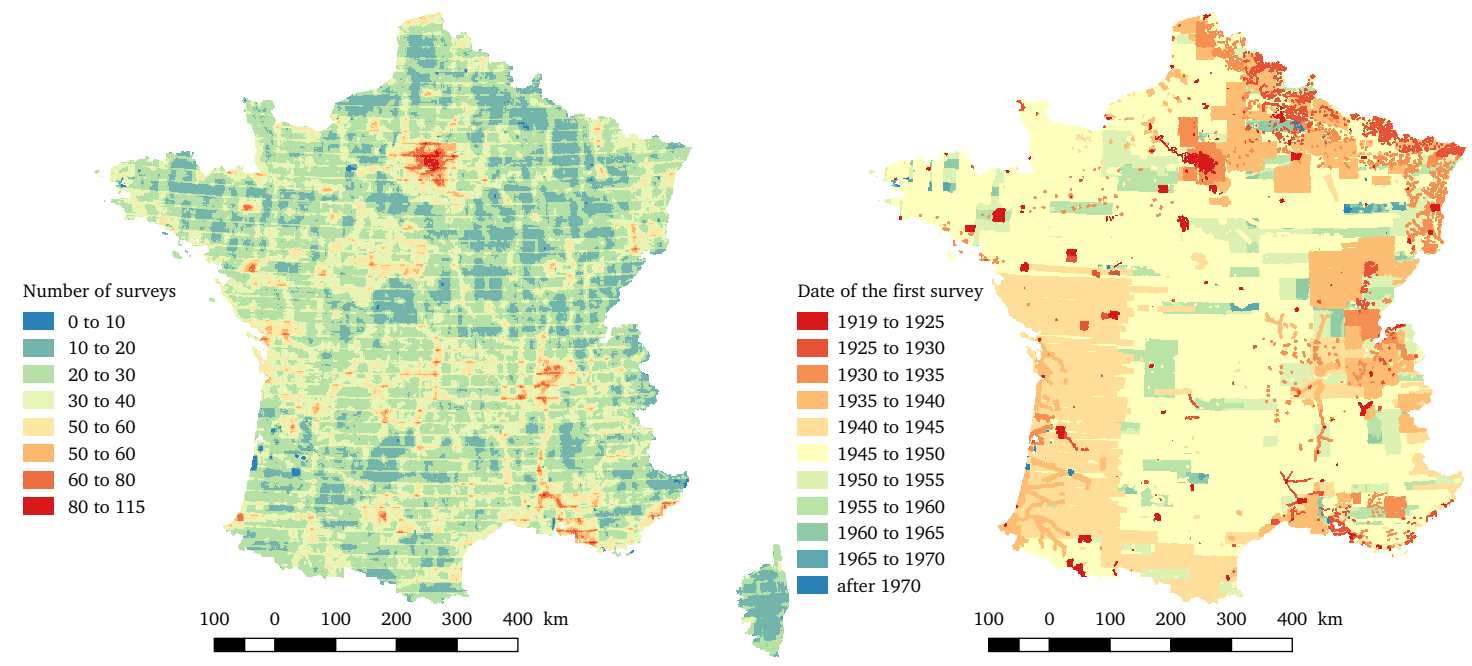

FIGURE 1. Cartes des prises de vues d'archives disponibles en France métropolitaine (2016) : nombre de missions (gauche) et date des plus anciennes acquisitions (droite). La maille de calcul est kilométrique.

Ces données d'archive constituent un moyen unique et peu exploité d'étudier le suivi des paysages sur le long terme et d'analyser les changements d'occupation du sol au cours du siècle passé (Giordano et al., 2017). Elles constituent en effet des séries temporelles (i) longues (les premières campagnes datent des années 1920), (ii) assez denses temporellement (avec généralement une campagne au moins tous les 2 à 5 ans), (iii) acquises avec une très haute résolution spatiale $(<1-2 \mathrm{~m}$ ) et, (iv) permettant généralement d'en déduire une information tridimensionnelle par des méthodes photogrammétriques. L'utilisation de ces données par des processus d'analyse d'images de télédétection nécessite toutefois un géoréférencement suffisamment fin pour être en mesure de générer des ortho-images et des Modèles Numériques de Surfaces (MNS) de qualité suffisante et permettre leur comparaison automatique à des dates différentes. Or, les images numériques diffusées aujourd'hui ont été créées à partir des clichés argentiques d'archive dont la mise en géométrie fine n'a pas été conservée : le plus souvent seule une information de localisation approchée de chaque cliché a été conservée tandis que le certificat d'étalonnage exact de la caméra utilisée pour la prise de vues n'est plus disponible. Toutefois, les métadonnées généralement diffusées avec les images comportent des informations utiles comme la taille du capteur ou sa focale, voire plus rarement son certificat d'étalonnage. Les tableaux d'assemblage et plans de vol de ces campagnes de prises de vues sont généralement fournis avec les images et apportent une géolocalisation approchée des clichés que l'on peut alors exploiter.

Cette étude concerne l'automatisation de la remise en géométrie (géoréférencement) de ces prises de vues d'archive. L'approche proposée permet, uniquement à partir de (méta-)données initiales assez grossières et sans intervention manuelle, la production d'ortho-images et de Modèles Numériques de Surface (MNS) de qualité compatible avec une comparaison multi-dates. Elle ouvre dé- sormais la porte à des exploitations thématiques des images aériennes historiques. Cet article est une extension de (Giordano et al., 2018).

\section{Géoréférencement fin de prises de vues anciennes}

Dans ce travail, on pose la condition qu'une localisation même approchée $(\sim 100 \mathrm{~m})$ de chaque cliché d'archive est disponible. De ce fait, on ne va ensuite s'intéresser qu'au géoréférencement fin de ces données dans un système cartographique donné. On vise une précision à même de permettre une comparaison des produits (radiométrie, hauteur, classes) générés à partir de ces campagnes d'archives à différentes dates.

On peut distinguer pour cette tâche de géoréférencement les approches non photogrammétriques et photogrammétriques. Les approches non photogrammétriques recherchent une transformation empirique recalant au mieux les images, tandis que les approches photogrammétriques visent à modéliser les paramètres de prise de vues de toutes les images, autorisant de ce fait le calcul de MNS.

La Figure 2 propose un aperçu global des approches rencontrées dans la littérature selon les caractéristiques spatiales et temporelles des campagnes aériennes d'archive traitées. Les caractéristiques considérées pour chaque étude sont : la surface des zones traitées, le nombre de dates ainsi que l'intervalle de temps considéré (i.e., la longueur de la série temporelle). On constate en premier lieu que la plupart de ces études reposent sur des approches photogrammétriques ( 6 non photogrammétriques contre 16 photogrammétriques) mais qu'aucune solution automatique n'a été proposée jusqu'alors. Les approches photogrammétriques recensées concernent majoritairement moins de 5 dates et moins de $100 \mathrm{~km}^{2}$ (Meyer et al., 1996; Kadmon et Harari-Kremer, 1999; Walstra et al., 2004; Cardenal et al., 2006; Fox et Cziferszky, 
2008; Mondino et Chiabrandoa, 2008; Véga et St-Onge, 2008; Ayoub et al., 2009; Aguilar et al., 2013; Necsoiu et al., 2013; Nebiker et al., 2014). (Micheletti et al., 2015) (9 dates), (Nurminen et al., 2015) (10) et (Korpela, 2006) (28) sont les seuls travaux pour lesquels plus de 5 millésimes ont été traités. Dans (Nurminen et al., 2015), des zones relativement plus étendues sont abordées $\left(600 \mathrm{~km}^{2}\right)$ pour générer des MNS dans des zones de forêt, sans en faire toutefois une approche généralisable pour $d$ eplus grandes surfaces. Des conclusions identiques (nombres de dates et surfaces limitées) peuvent être énoncées pour les approches non photogrammétriques (Ellis et al., 2006; Jao et al., 2014; Chen et al., 2016), bien que certains de ces travaux se soient efforcés de traiter des zones plus étendues (Asner et al., 2003; Galster et al., 2008; Ford, 2013). Enfin, la période considérée et l'âge des clichés ne sont pas nécessairement des facteurs limitants : la longueur moyenne des séries temporelles est de 30 ans et la première date traitée se situe souvent dans la période 1945-1955. Ces limitations à la fois spatiales (taille de la zone traitée) et temporelles (nombre de dates traitées) sont dues à plusieurs facteurs. Le plus contraignant concerne l'important volume de travail opérateur au cours du processus de géoréférencement.

\subsection{Approches non photogrammétriques}

Les techniques non photogrammétriques mises en œuvre pour géoréférencer finement des prises de vues anciennes consistent généralement en une simple transformation polynomiale appliquée à chaque cliché. La détermination des paramètres de ces transformations nécessite de disposer de références (amers) terrain (généralement des points d'appui). Elles sont issues d'orthoimages satellitaires (Ellis et al., 2006; Ford, 2013) ou aériennes récentes issues de bases de données nationales (Ellis et al., 2006; Galster et al., 2008) ou de campagnes de mesures terrain, par exemple des mesures GPS de centres de carrefours routiers comme dans (Asner et al., 2003). Ces approches requièrent un équipement dense d'amers terrain pour chaque cliché de la prise de vues. Ainsi, selon les travaux, on dénombre au moins 6 points d'appui par cliché pour (Galster et al., 2008), 20 points par cliché pour (Ellis et al., 2006), entre 10 et 47 pour (Ford, 2013), et 56 pour (Asner et al., 2003). Le modèle de recalage peut par exemple être une affinité 2D (Galster et al., 2008) ou une transformation polynomiale (Ford, 2013). La précision planimétrique atteinte est généralement considérée comme compatible avec une analyse multi-dates ( $<1 \mathrm{~m}$, (Asner et al., 2003; Galster et al., 2008) - <2 m (Ford, 2013)). La détermination d'amers communs entre jeux de données récents et anciens reste toutefois une tâche complexe, en particulier dans les zones en forte évolution. Certains auteurs, comme (Chen et al., 2016), estiment même que cette difficulté rend impossible le traitement de toutes ces prises de vues d'archive. Par ailleurs, certains travaux (Jao et al., 2014; Chen et al., 2016) utilisent des techniques classiques de détection de points homologues (comme celle de (Lowe, 1999)). II ne s'agit pas de les détecter entre les clichés anciens et une référence image récente mais plus simplement d'identifier des points de liaison entre clichés anciens afin de réduire le nombre d'amers terrain nécessaires à l'estimation des paramètres de la transformation. Ainsi, Jao et al. (2014) estiment les paramètres de chaque transformation ( 6 paramètres pour une affinité dans ce cas) en utilisant des points de liaison entre les clichés de la prise de vues d'archive. Ils peuvent donc se contenter d'un nombre plus restreint de points d'appui ( 6 points pour 6 clichés) sur l'ensemble du bloc d'images. Les précisions planimétriques obtenues demeurent toutefois insuffisantes $(17 \mathrm{~m})$. La même approche combinant points de liaison automatiques entre clichés anciens et points d'appui manuels permet dans (Chen et al., 2016) d'obtenir une précision planimétrique de $1 \mathrm{~m}$ pour une homographie à 8 paramètres calculée à partir de 20 points d'appui pour 12 images.

Les approches non photogrammétriques permettent d'obtenir, à partir de clichés anciens, des ortho-images géoréférencées pour une précision planimétrique acceptable. Toutefois, même si quelques améliorations ont pu être proposées, la nécessaire intervention d'un opérateur constitue une limitation majeure à leur application à plus de dates ou à des zones plus étendues. L'extraction de points de liaison entre clichés anciens et images actuelles géoréférencées mériterait d'être étudiée. L'absence de géoréférencement approché des images anciennes ainsi que les changements d'occupation du sol parfois importants constituent les principales difficultés.

\subsection{Approches photogrammétriques}

Les approches non photogrammétriques vues précédemment permettent de produire des ortho-images géoréférencées à partir des prises de vues d'archives. En revanche, contrairement aux approches photogrammétriques, elles ne permettent pas d'accéder à une information tridimensionnelle.

De nombreuses chaînes logicielles de photogrammétrie existent et sont capables de traiter d'anciens chantiers de prises de vues argentiques (Pierrot Deseilligny et Cléry, 2011; Nebiker et al., 2014). Ces outils mettent généralement en œuvre un processus classique : orientation interne, mise en place relative puis absolue (aérotriangulation) et enfin production de MNS et d'orthoimage. Dans la suite, on s'intéresse au niveau d'automatisation proposé par plusieurs de ces outils pour chaque étape.

\subsubsection{Orientation interne}

L'orientation interne d'un cliché argentique sert à corriger les déformations subies par son film au cours de la prise de vues et du développement ainsi que les distorsions créées lors de sa numérisation. II s'agit d'une transformation mathématique (généralement une affinité 


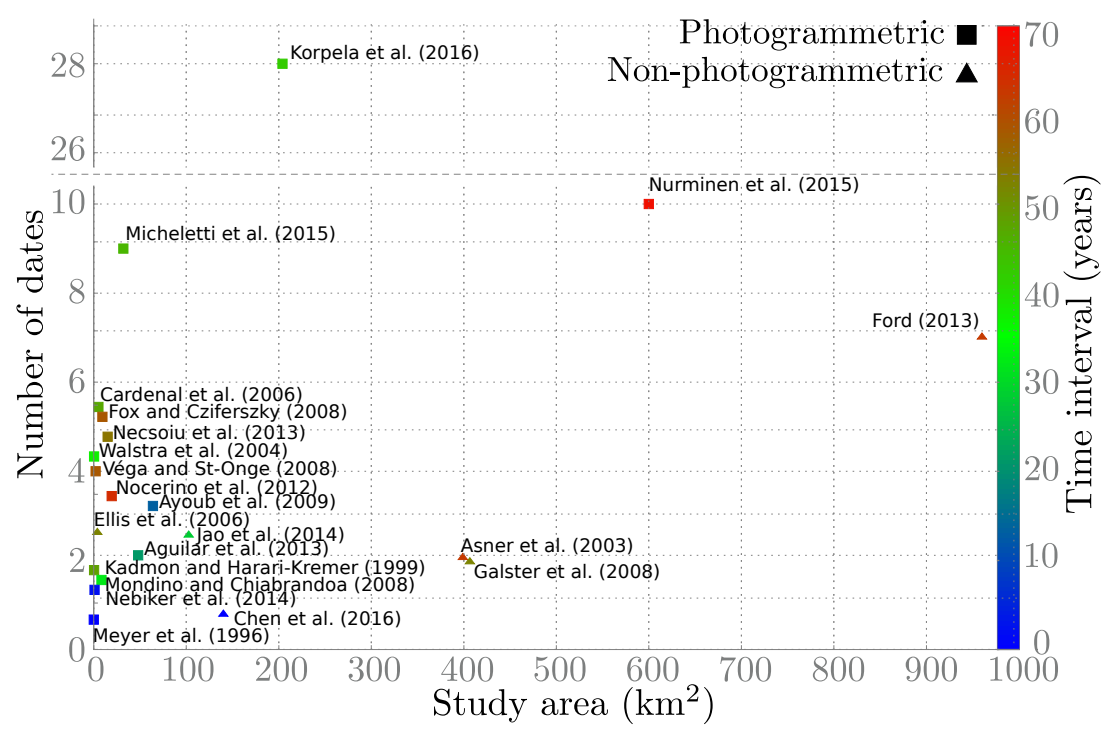

FIgURE 2. Vue d'ensemble des travaux mentionnés dans l'état de l'art. Les différents articles sont positionnés selon la taille de la zone d'étude, le nombre de dates et la longueur des séries temporelles traitées. Ils sont également catégorisés selon leur type d'approche.

2D) entre le repère associé au fond de chambre de la caméra et le repère image de chaque cliché. Les repères de fond de chambre servent alors de références pour ce calcul. Presque tous les travaux recensés dans cet état de l'art utilisent des images aériennes comportant des repères de fond de chambre. Les certificats d'étalonnage peuvent permettre de retrouver les coordonnées de ces repères dans le référentiel caméra (Korpela, 2006; Véga et St-Onge, 2008; Micheletti et al., 2015; Nurminen et al., 2015). Toutefois, ils ne sont pas toujours facilement disponibles, ce qui peut constituer un premier obstacle au traitement de plusieurs dates. Aussi, certains travaux proposent d'utiliser une information approchée. Aguilar et al. (2013) ne se servent que de l'information sur la focale qui est généralement conservée ou apparaît directement sur les clichés, tandis que Nocerino et al. (2012) se passent de certificats de calibration en retrouvant une information approchée directement à partir des images. Les auteurs ne mentionnent généralement pas la manière dont ils mesurent les coordonnées image des repères de fond de chambre. De nombreuses solutions logicielles (comme par exemple MicMac (Pierrot Deseilligny et Cléry, 2011)) proposent une détection semi-automatique des repères de fond de chambre afin de retrouver leurs coordonnées images dans les clichés. La saisie manuelle des repères de fond de chambre sur une image de la prise de vues reste toutefois nécessaire pour initialiser le processus.

\subsubsection{Mise en place relative - aérotriangulation "en l'air"}

La mise en place relative des clichés s'effectue de manière totalement automatique : une aérotriangulation dite "en l'air" est calculée à partir de points de liaison entre clichés détectés par un processus automatique. Les paramètres internes de la caméra sont générale- ment ré-estimés au cours de ce calcul par auto-étalonnage (Walstra et al., 2004; Cardenal et al., 2006; Fox et Cziferszky, 2008; Necsoiu et al., 2013; Nurminen et al., 2015). II convient toutefois de garder à l'esprit que les résultats de cet auto-étalonnage restent le plus souvent une approximation du fait des configurations peu contraintes de ces prises de vues. Cette mise en place relative des images a un impact important sur les précisions planimétriques et altimétriques des ortho-images et MNS générés dans la suite. Aussi, certains auteurs interviennent manuellement au cours de cette étape. Ils rajoutent quelques points de liaison mesurés par un opérateur (Micheletti et al., 2015), qui peuvent même être des points de liaison inter-dates lorsque plusieurs dates sont orientées simultanément (Cardenal et al., 2006).

\subsubsection{Mise en place absolue}

La mise en géométrie des clichés en absolu dans un système cartographique nécessite de disposer de références de coordonnées terrain connues. La méthode la plus commune consiste à utiliser comme amers des points d'appui, pointés manuellement dans les images. Tout comme dans le cas des approches non photogrammétriques, les coordonnées terrain de ces points sont issues de mesures manuelles sur une ortho-image et un MNS de référence (Ellis et al., 2006; Fox et Cziferszky, 2008; Véga et St-Onge, 2008; Nocerino et al., 2012; Necsoiu et al., 2013; Nurminen et al., 2015), de campagnes de mesures GPS sur le terrain (Walstra et al., 2004; Cardenal et al., 2006; Micheletti et al., 2015), ou d'une combinaison des deux (Aguilar et al., 2013). Le nombre de points d'appui nécessaires est important par rapport à la taille des sites étudiés : près de 45 points pour moins de 10 clichés dans (Nurminen et al., 2015) ou (Aguilar et al., 2013), ou 169 point pour une zone de $20 \mathrm{~km}^{2}$ dans 
les travaux de (Micheletti et al., 2015), de 10 à 47 points par image dans (Ford, 2013). Ce nombre important de points d'appui est nécessaire du fait de l'absence à la fois d'informations sur l'étalonnage de la caméra et de valeurs initiales de l'orientation externe des clichés suffisamment précises (Nurminen et al., 2015). Aussi, on procède généralement à un nouvel auto-étalonnage de la caméra au cours de ce calcul d'aérotriangulation.

A notre connaissance, aucune étude sur l'automatisation de la prise des points d'appui n'a été proposée. Or, tous les travaux soulignent que leur détermination est longue et fastidieuse. En effet, outre le fait de devoir les pointer, déterminer ces points est souvent difficile : il s'agit en effet d'une part d'identifier des détails à la fois pérennes sur une période potentiellement longue et d'autre part identifiables avec précision sur les clichés (Micheletti et al., 2015). La précision planimétrique et altimétrique de la mise en géométrie absolue des images dépend en particulier de l'échelle ( $\sim$ résolution spatiale) de la prise de vues. La plupart des études obtiennent, seIon l'échelle des prises de vues, des précisions planimétriques de $<1$ m à 2 m (Cardenal et al., 2006; Véga et StOnge, 2008; Fox et Cziferszky, 2008; Aguilar et al., 2013; Necsoiu et al., 2013; Micheletti et al., 2015) ou entre $2 \mathrm{~m}$ to $10 \mathrm{~m}$ (Nocerino et al., 2012). Pour toutes ces raisons, cette étape de remise en géométrie en absolu reste le principal verrou pour une automatisation complète des traitements, ou tout au moins pour une limitation des interventions opérateur.

Peu de méthodes proposent des solutions pour dépasser ces limites. Korpela (2006) effectue une aérotriangulation multi-dates impliquant des images récentes d'orientation absolue connue. Ce traitement nécessite toutefois l'identification manuelle de points de liaison interdates (279 points pour 288 images). Cléry et al. (2014) et Nagarajan et Schenk (2016) proposent de remplacer les points d'appui comme référence terrain par des primitives linéaires correspondant à des structures pérennes comme les axes des routes ou les contours des bâtiments. La saisie manuelle de ces primitives linéaires, à la fois dans les clichés anciens et la donnée de référence, reste nécessaire pour (Nagarajan et Schenk, 2016). En revanche, Cléry et al. (2014) effectuent une mise en correspondance automatique des objets d'une base de donnée topographique $2 \mathrm{D}$ avec les lignes détectées dans les images est effectué. Cette méthode n'a toutefois été appliquée que pour orthorectifier les clichés en les redressant indépendamment les uns des autres : aucune compensation globale n'a été effectuée sur un bloc d'images plus conséquent.

\subsubsection{MNS et ortho-images}

Une fois les images mises en géométrie (poses externes calculées et caméra étalonnée), il est possible d'en dériver des ortho-images et des MNS. Le MNS est calculé par appariement dense (Pierrot Deseilligny et Cléry, 2011). II peut ensuite servir à orthorectifier les différents clichés de la prise de vues, et même à pro- duire des ortho-images vraies. II s'agit alors de fusionner ces différents clichés orthorectifiés en une ortho-image unique couvrant l'intégralité de la zone d'intérêt. Il peut aussi être utile d'effectuer une égalisation radiométrique des images au cours de ce processus de mosaïquage. Toutefois, parmi les papiers présentant le traitement de prises de vues anciennes, peu de travaux s'intéressent à cette étape.

Des MNS ont été produits à partir de prises de vues anciennes dans (Walstra et al., 2004; Cardenal et al., 2006; Véga et St-Onge, 2008; Nocerino et al., 2012; Nurminen et al., 2015; Giordano et al., 2017). Le nombre important de points d'appui utilisés dans ces travaux semble permettre d'obtenir des précisions altimétriques de l'ordre du mètre (Cardenal et al., 2006; Nurminen et al., 2015) jusqu'à $4 \mathrm{~m}$ (Walstra et al., 2004). Cette précision altimétrique dépend notamment de l'échelle des clichés. Un appariement dense peut toutefois être appliqué aux clichés d'une ancienne prise de vues aérienne stéréoscopique pour peu que la qualité de leur mise en géométrie est suffisante. Un comparatif selon différents critères de 3 méthodes d'appariement dense pour les clichés anciens est présenté dans (Nebiker et al., 2014).

\subsection{Approche proposée}

II ressort de l'état de l'art précédent que la détermination d'un équipement en amers de référence terrain (appuis) suffisant constitue le principal obstacle au traitement des prises de vues photogrammétriques anciennes sur des zones plus étendues et davantage de dates. Ce problème concerne à la fois les approches photogrammétriques et non photogrammétriques. Au vu de l'état de l'art précédent, très peu de travaux ont été consacrés à automatiser cette tâche.

Cette étude vise donc à proposer un processus automatique outrepassant les problèmes mentionnés précédemment. L'approche proposée est photogrammétrique : on considère en effet que la remise en géométrie des images ne doit pas se limiter à une simple orthorectification, mais doit également permettre d'accéder à une information tridimensionnelle par le calcul d'un MNS. Un tel MNS est nécessaire dans de nombreux applications thématiques. La stratégie mise en œuvre cherche à tirer pleinement parti des informations approchées sur la localisation des clichés et l'étalonnage de la caméra fournies par les métadonnées des clichés : on part d'une solution approchée que l'on cherche à affiner par rapport à un jeu de données de référence récentes. En particulier, une solution est proposée afin de déterminer automatiquement des amers de référence terrain suffisamment denses et stables au cours du temps.

A notre connaissance, la recherche automatique de points homologues entre deux acquisitions distinctes n'a jamais été mise en œuvre pour le traitement des prises de vues aériennes anciennes. On est en effet alors confronté à la fois au fait que ces deux acquisitions peuvent être séparées par une période suffisamment longue pour 
que d'importants changements de l'occupation du sol se soient produits et au fait que les images de ces deux missions peuvent être très différentes (en termes de radiométrie et de résolution spatiale).

Ces difficultés sont ici surmontées au cours d'un processus de géoréférencement absolu des images en deux étapes.

1. Une ortho-image et un MNS "grossiers" sont d'abord produits pour chaque millésime sans données de référence terrain (hormis les métadonnées basiques généralement fournies avec les clichés).

2. Les clichés anciens sont ensuite mis en géométrie de manière plus fine à l'aide de points d'appui détectés automatiquement entre ces orthoimages "grossières" et un jeu de référence récent constitué d'une ortho-image et d'un MNS (permettant donc de déterminer les coordonnées terrain tridimensionnelles de ces points d'appui).

L'orientation absolue approximative des clichés anciens ainsi que le MNS "grossier" calculés précédemment sont utilisés afin de déterminer automatiquement les coordonnées de ces points d'appui dans les clichés anciens (mesures clichés de ces points d'appui).

\section{Méthodes mises en œuvre}

La figure 3 présente la chaîne de traitements proposée, avec ses principales entrées et sorties. On commence par les traditionnelles étapes d'orientations interne et relative (mise en géométrie en l'air) qui reposent sur la mise en œuvre de méthodes photogrammétriques classiques de la littérature (Section 3.2). Ensuite, une étape de mise en géométrie absolue originale en 2 étapes (géoréférencement grossier, puis fin) est proposée (Sections 3.3 et 3.5). Elle comporte un calcul automatique de points d'appui qui fournit des amers terrain pour une nouvelle aérotriangulation (Section 3.4).

\subsection{Données d'entrée}

Les données nécessaires à la méthode proposée sont (i) les clichés anciens numérisés, (ii) accompagnés de métadonnées très basiques. Ces métadonnées se rapportent au processus de numérisation (résolution du scan) et à la configuration de la prise de vues (focale de la caméra, dimensions physiques $(\mathrm{mm})$ des clichés et plan de vol approché). La focale et les dimensions des clichés ont généralement été conservées. Un plan de vol approché est également nécessaire, mais n'a pas besoin d'être très précis. Des valeurs approchées $(\approx 100 \mathrm{~m})$ des coordonnées terrain planimétriques du nadir des images sont disponibles dans la plupart des cas, en tant que données de base nécessaires à la diffusion des images par le biais de services en ligne. La connaissance d'une valeur approximative de la hauteur de vol est également nécessaire, afin de disposer d'une valeur approchée de

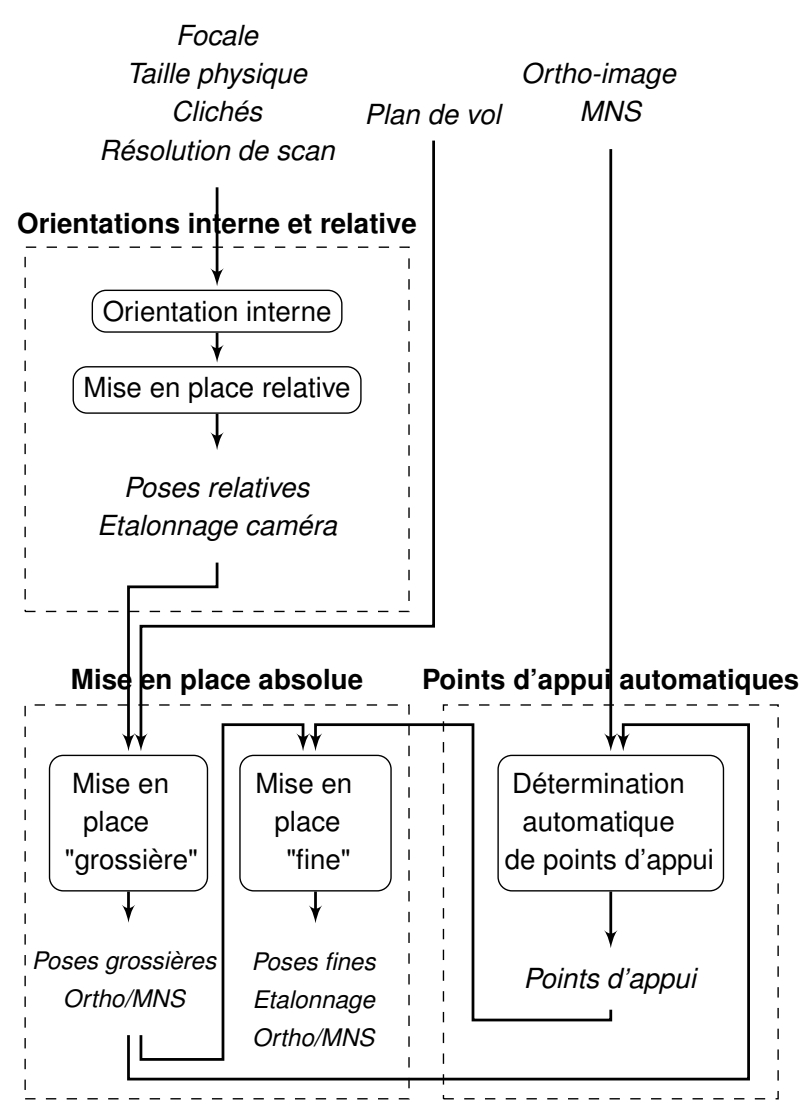

FIGURE 3. Approche proposée (voir le texte pour plus de détails).

la composante altimétrique des coordonnées terrain des sommets de prise de vues des clichés.

On a également besoin d'ortho-images et de MNS récents. Ces données sont en effet utilisées comme des références planimétriques et altimétriques par rapport auxquelles les clichés anciens sont orientés. Dans le cas présent, ces données sont issues des bases de données nationales de I'IGN (notamment la BDOrtho pour les ortho-images).

\subsection{Orientation interne et mise en place relative}

Pour ces étapes d'orientation interne et de mise en place relative des clichés (processus dit "en l'air"), la chaîne de traitement proposée met en œuvre des méthodes de l'état de l'art généralement implémentées dans les logiciels de photogrammétrie. Plus précisément, l'orientation interne est d'abord effectuée à l'aide d'une méthode semi-automatique implémentée dans la suite logicielle MicMac (Pierrot Deseilligny et Cléry, 2011). Les coordonnées image des repères de fond de chambre sont d'abord pointés à la main sur un cliché ancien, puis retrouvés automatiquement sur les autres clichés de la même prise de vues. Les images sont ensuite ré-échantillonnées selon leur orientation interne et une mise en place relative "en l'air" des clichés est alors calculée à partir de points de liaison détectés automatiquement dans les dif- 
férents clichés. Cette aérotriangulation (calculée par compensation par faisceaux) intègre également un auto-étalonnage de la caméra.

\subsection{Mise en place absolue "grossière"}

Cette première mise en place absolue consiste ici à calculer une transformation géométrique (une similitude 3D) pour passer de la mise en place relative des clichés à une mise en place absolue, c'est-à-dire pour passer du repère "en l'air' de la mise en place relative au repère terrain. Les paramètres de cette transformation sont estimés à partir d'une part des valeurs approchées fournies par le plan de vol pour les coordonnées terrain des sommets de prise de vues des clichés, et d'autre part de leurs coordonnées dans la mise en place "en l'air". On obtient alors une pose approchée des clichés en absolu. On calcule ensuite des MNS et des ortho-images (vraies) à partir de ces poses. Dans la suite, on y fait référence par les termes d'ortho-images et de MNS "grossiers".

\subsection{Détermination automatique d'amers terrain}

La littérature comporte plusieurs approches dédiées à la détection et la mise en correspondance de points homologues entre données de télédétection multi-modales. On peut par exemple mentionner les méthodes utilisant le descripteur DASC "Dense Adaptive Self-Correlation" (Kim et al., 2015). A notre connaissance, ces méthodes n'ont toutefois pas été appliquées au cas fortement diachronique des chantiers de prises de vues aériennes d'archive.

Certaines méthodes ont été développées spécifiquement afin d'être plus robustes et plus efficaces pour détecter des points homologues entre images diachroniques. Elles consistent notamment souvent simplement à appréhender des éclairages différents. Les approches mises en œuvre reposent alors souvent sur des techniques d'apprentissage : des modèles de détection et de mise en correspondance de points d'intérêt sont alors définis à l'issue d'un apprentissage à partir d'exemples de référence. Parmi ces méthodes, TILDE (Verdie et al., 2015) formule ce problème sous la forme d'un modèle de régression tandis que LIFT (Yi et al., 2016) adopte un modèle d'apprentissage profond.

Dans ce travail, c'est l'approche proposée dans (Aubry et al., 2014) qui a été retenue. Cet algorithme a en effet été développé spécifiquement afin de détecter des points homologues entre des images et des peintures historiques. Pour cette raison, on peut attendre de cet algorithme qu'il soit en mesure de traiter des données à la fois multi-dates et multi-modales. Cette méthode utilise des descripteurs de type histogrammes de gradients ou HoG (Dalal et Triggs, 2005) et considère également la mise en correspondance de ces descripteurs comme un problème de classification. L'idée sous-jacente est alors d'entraîner pour le descripteur HoG de chaque pixel un classifieur binaire "descripteur similaire" / "descripteur différent" à partir d'un seul échantillon positif et d'un nombre important d'exemples négatifs. L'algorithme comporte deux étapes distinctes : la détection de points d'intérêt et la mise en correspondance de ces points.

\subsubsection{Détection de points d'intérêt}

Une approche multi-résolutions est mise en œuvre lors de la détection des points d'intérêt. On commence par construire un espace d'échelles à partir de l'image. Pour chaque pixel d'un niveau donné de l'espace d'échelles, on calcule un score mesurant à quel point son descripteur HoG est discriminant. Evaluer si un descripteur est discriminant revient ici à mesurer la difficulté d'entrấner un classifieur de type Séparateur à Vastes Marges (SVM) linéaire pour une fonction de perte (loss) quadratique à décider si un autre descripteur lui est "similaire" ou "différent". Le score est donc directement lié au coût de l'entraînement de ce classifieur. Les points d'intérêt sont alors détectés à chaque niveau de cet espace d'échelles comme étant les maxima locaux de ce score.

En pratique, les points d'intérêt sont détectés dans les ortho-images récentes utilisées comme références. Comme ces ortho-images sont géoréférencées, il est possible d'associer directement des coordonnées terrain planimétriques à ces points d'intérêt. L'information sur leur composante altimétrique est ensuite directement récupérée depuis le MNS de référence. On obtient donc directement des points terrain tridimensionnels.

\subsubsection{Mise en correspondance}

Les points d'intérêt détectés précédemment sont ensuite mis en correspondance avec l'ortho-image "grossière" calculée à partir des clichés anciens au cours des étapes précédentes. On adopte une approche de traitement par dalle. Le géoréférencement approché de cette ortho-image "grossière" permet en effet de disposer d'un prédicteur. Aussi, pour chaque dalle d'ortho-image ancienne, on ne sélectionne parmi les points d'intérêt détectés dans l'ortho-image de référence que le sous-ensemble de points susceptibles d'avoir un homologue au sein de cette dalle. On procède alors à une mise en correspondance dense : un espace d'échelles est construit à partir de la dalle d'ortho-image ancienne, et des scores d'appariement sont calculés entre le descripteur HoG de chaque point d'intérêt et celui de chaque pixel de chaque niveau de cet espace d'échelles.

Ce score d'appariement est défini en formulant cette fois le problème de classification binaire "descripteur similaire" / "descripteur différent" comme une Analyse Discriminante Linéaire. Pour chaque point d'intérêt issu de la donnée de référence, on s'intéresse aux deux meilleurs scores d'appariement obtenus. A la manière de (Lowe, 1999), on calcule le ratio entre les scores de ces deux meilleurs candidats afin d'évaluer l'ambiguïté de la mise en correspondance, et finalement, on ne conserve que les meilleurs appariements au sens de ce critère. 
On applique ensuite un filtrage RANSAC à ces jeux de points mis en correspondance, afin d'éliminer les fautes les plus grossières. II s'agit ensuite de récupérer les coordonnées image dans les clichés anciens des points conservés à l'issue de ce filtrage. On exploite ici le fait que l'ortho-image ancienne est en réalité une ortho-image vraie et que l'on dispose également du MNS qui lui est associé. On récupère donc d'abord pour chacun des points ses coordonnées 3D à partir du jeu ortho-image / MNS "grossiers", et on leur applique les modèles de passage du terrain vers les images issus de la mise en place absolue "grossière".

\subsection{Mise en place absolue finale}

Cette dernière étape a pour but d'améliorer la mise en place absolue des clichés ainsi que l'étalonnage de la caméra. Cette nouvelle aérotriangulation est une compensation par faisceaux calculée par moindres carrés robustes. Elle intègre à la fois les points d'appui déterminés automatiquement en section 3.4 et les points de liaison de la section 3.2. La mise en place absolue approchée des clichés obtenue en 3.3 sert de solution initiale.

Enfin, des ortho-images (vraies) et des MNS "fins" sont calculés à partir des nouvelles poses des clichés obtenues à l'issue de ce calcul.

\section{Resultats}

\subsection{Jeux de données}

La méthode a été testée sur 2 zones d'études très différentes.

La zone de Fabas est une zone agricole et forestière au sein de laquelle les zones urbaines ou artificialisées ont peu évolué. Elle se situe dans le Sud-Ouest de la France (31). Les changements d'occupation du sol les plus importants correspondent au remembrement de parcelles agricoles, à la création d'un lac artificiel ainsi qu'à l'évolution de la forêt.

La zone d'étude de Fréjus se situe quant à elle dans le Sud-Est de la France (83). Cette zone a connu une forte urbanisation : principalement agricole avec de petits villages à la fin de la Seconde Guerre Mondiale, elle s'est progressivement urbanisée avec le développement à la fois de zones urbaines continues et denses, et de quartiers plus résidentiels.

Pour chaque zone d'étude, on a sélectionné 5 prises de vues aériennes distinctes (Tableau 1). Pour chaque mission, seules les images panchromatiques ont été testées.

Par ailleurs, la méthode mise en œuvre nécessite de disposer d'ortho-image et de MNS de référence. Ces données sont ici issues des bases de données nationales de référence de l'IGN. Les ortho-images proviennent de la BDOrtho : elles présentent une résolution spatiale de $0.5 \mathrm{~m}$ et ont été produites à partir d'images aériennes acquises respectivement en 2014 et en 2010 pour les zones de Fréjus et de Fabas. Les MNS, calculés par corrélation dense à partir de ces prises de vues aériennes, ont également une résolution spatiale de $0.5 \mathrm{~m}$.

\subsection{Evaluation des résultats}

\subsubsection{Ortho-images et MNS "grossiers"}

La figure 4 présente un exemple d'ortho-image et de MNS "grossiers" calculés sur une petite partie de la zone de Fabas à partir des clichés d'une prise de vues de 1984. Le niveau de détails du MNS est d'un simple point de visuel très informatif : routes, forêts et haies y apparaissent en effet très clairement sur cette zone naturelle. Les ortho-images "grossières", bien qu'issues du mosaïquage des différents clichés de la prise de vues, ne présentent pas véritablement de cisaillements locaux au niveau des transitions entre clichés. La comparaison entre les figures $4 a, b$ et $4 c, d$ montre que l'échelle de ces produits "grossiers" est cohérente avec la donnée de référence mais qu'il semble y avoir un biais pour le recalage planimétrique ( $\sim 120 \mathrm{~m}$ uniquement selon l'axe $\mathrm{Y}$ dans cet exemple). Un tel décalage s'observe aussi sur la figure 6 pour la mission de 1942. Une transformation polynomiale simple appliquée à cette ortho-image "grossière" et calculée à partir de quelques points d'appui issus de l'ortho-image de référence s'avère alors suffisante pour les corriger. Le résultat de cette correction correspond à l'ortho-image de fond de la figure 4c).

Néanmoins, la comparaison du MNS "grossier" au MNS récent de référence révèle l'existence d'écarts altimétriques importants et systématiques (figure 4c, mais aussi figure 9 pour une autre prise de vues), qu'il n'est cette fois pas possible de corriger par un modèle polynomial simple. Ce problème est vraisemblablement dû à la difficulté d'estimer à la fois l'étalonnage de la caméra et l'orientation des clichés exclusivement à partir de points de liaison, dans des configurations peu favorables (images visant le nadir et recouvrement notamment latéral peu important).

\subsubsection{Ortho-images et MNS "fins"}

La figure 5 présente quelques exemples de points d'appui détectés automatiquement (Fréjus). Dans cet exemple, l'ortho-image de référence date de 2014 et les clichés anciens de 1954. On peut constater que tous les points détectés n'ont pas la même précision, mais que même les moins bons peuvent généralement constituer des points d'ancrage même approchés susceptibles d'améliorer le géoréférencement approché des données. On peut aussi remarquer que ces points d'appui sont le plus souvent détectés au voisinage de structures artificielles (routes et bâtiments), ce qui présente un risque de densité inégale de ces points en particulier dans des zones naturelles.

Ces points d'appui sont ensuite utilisés dans un calcul d'aérotriangulation (compensation par faisceaux résolue par moindres carrés robustes afin de tenir compte des fautes). Le tableau 2 présente le nombre de points d'appui actifs ainsi que la moyenne des valeurs absolues 


\begin{tabular}{|c|c|c|c|c|c|c|c|}
\hline Date & $\mathrm{nb}$ & échelle & $\begin{array}{l}\text { scan } \\
\text { (dpi) }\end{array}$ & $\begin{array}{l}\text { pixel sol } \\
(\mathrm{m})\end{array}$ & $\begin{array}{l}\text { focale } \\
(\mathrm{mm})\end{array}$ & $\begin{array}{c}\text { hauteur } \\
\text { (m) }\end{array}$ & $\begin{array}{c}\text { taille } \\
(\mathrm{mm} \times \mathrm{mm})\end{array}$ \\
\hline & \multicolumn{7}{|c|}{ Fabas $(12 \mathrm{~km} \times 10 \mathrm{~km})$} \\
\hline 1942 & 55 & 20000 & 768 & 0.66 & 150 & 3400 & $180 \times 130$ \\
\hline 1962 & 35 & 25000 & 1200 & 0.53 & 125 & 3425 & $180 \times 180$ \\
\hline 1971 & 25 & 30000 & 1200 & 0.64 & 152 & 4800 & $230 \times 230$ \\
\hline 1984 & 36 & 17000 & 1200 & 0.36 & 214 & 3870 & $230 \times 230$ \\
\hline \multirow[t]{2}{*}{1992} & 23 & 25000 & 1200 & 0.53 & 153 & 3900 & $230 \times 230$ \\
\hline & \multicolumn{7}{|c|}{ Fréjus $(3 \mathrm{~km} \times 2 \mathrm{~km})$} \\
\hline 1954 & 19 & 5000 & 1200 & 0.11 & 502 & 2530 & $300 \times 300$ \\
\hline 1966 & 15 & 8000 & 1200 & 0.17 & 210 & 1780 & $180 \times 180$ \\
\hline 1970 & 19 & 8000 & 1200 & 0.17 & 210 & 1770 & $180 \times 180$ \\
\hline 1978 & 10 & 14500 & 1200 & 0.31 & 153 & 2315 & $230 \times 230$ \\
\hline 1989 & 12 & 10000 & 1200 & 0.21 & 214 & 2550 & $230 \times 230$ \\
\hline
\end{tabular}

TABLE 1. Prises de vues traitées pour chacune des deux zones d'étude. $n b$ : nombre de clichés de la prise de vues; scale: échelle cartographique de la prise de vues; scan : résolution de scan lors de la numérisation; pixel sol : estimation de la taille du pixel sol ; focale : focale de la caméra; hauteur : hauteur de vol ; taille : dimensions physiques des clichés.

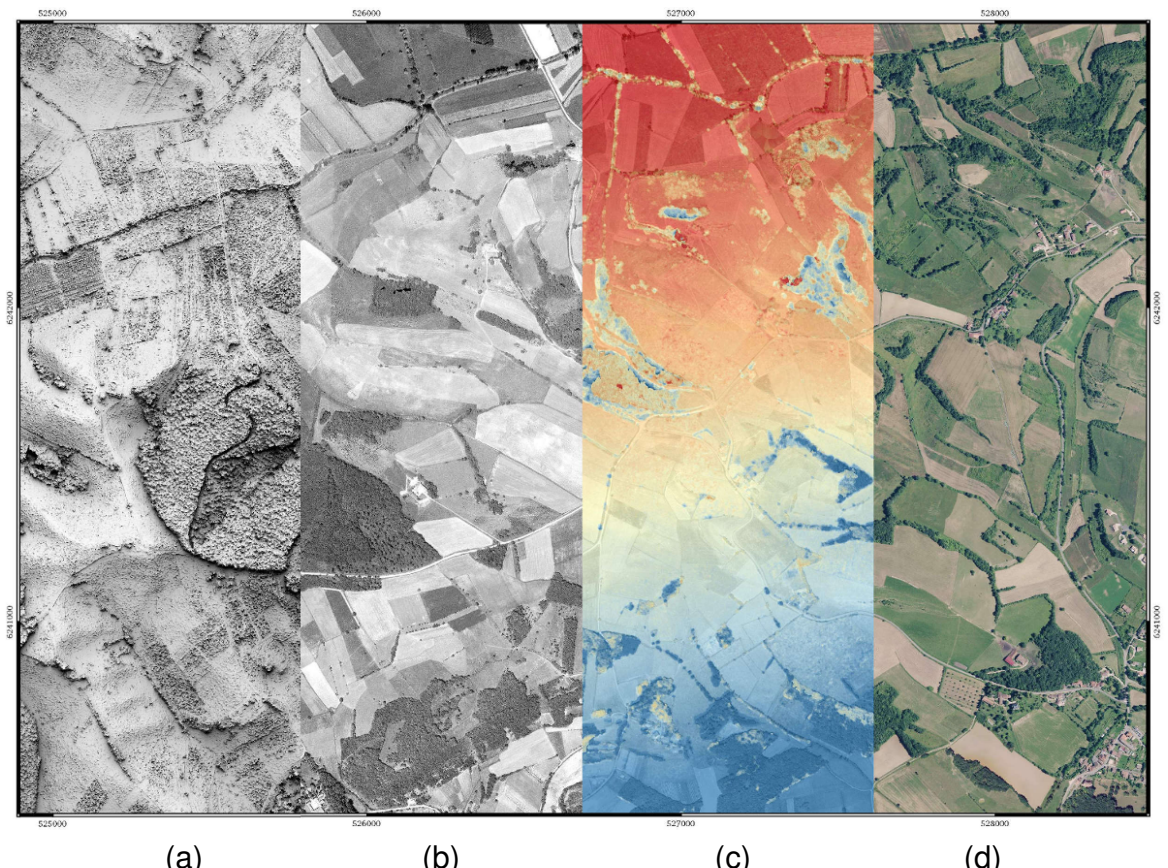

(a)

(b)

(c)

(d)

FIGURE 4. Exemples de résultats pour Fabas 1984 (3,5 km×3 km). (a) MNS "grossier"; (b) Ortho-image "grossière"; (c) Erreurs altimétriques entre le MNS "grossier" et le MNS de référence récent $(-60 \mathrm{~m} \rightarrow+20 \mathrm{~m})$; (d) Ortho-image récente utilisée comme référence.

des résidus sur ces points à l'issue du calcul.

Cette évaluation quantitative demeure toutefois insuffisante. Elle repose en effet uniquement sur les résidus des points d'appui. Or, il convient de garder à l'esprit que ces points détectés automatiquement présentent une qualité variable. En effet, même dans le cas d'appariements corrects, leur précision planimétrique dépend du niveau de l'espace d'échelle auquel ils ont été détectés. Par ailleurs, leurs coordonnées terrain planimétriques sont calculées à partir d'une ortho-image non vraies qui peuvent présentées des décalages 2D dus au relief. De même, leur composante altimétrique est estimée à partir d'un MNS de référence. Sa qualité est donc liée à celle de ce MNS. Ainsi, des résidus assez importants sur un de ces points d'appui peuvent en réalité correspondre à une réalité (comme par exemple un changement d'occupa- tion du sol) et non à une mauvaise prise en compte de ce point dans la compensation par faisceaux. Une évaluation plus rigoureuse des précisions planimétriques et altimétriques sera effectuée à brève échéance. Elle reposera sur des points de contrôle 3D mesurés par un opérateur directement à partir des clichés.

Les ortho-images "fines" calculées à partir des anciennes prises de vues ont d'abord été évaluées visuellement sur les deux zones tests : on les a affichées conjointement avec les ortho-images de référence sous forme de damiers afin de vérifier la continuité et l'alignement d'éléments pérennes du paysage. Dans le même but, on a également superposé à ces ortho-images les objets bâtiments et réseaux routiers de la BDTopo de l'IGN. Les figures 7 et 8 présentent pour les deux zones d'étude des exemples représentatifs de ces vues. Le recalage plani- 
métrique entre ces ortho-images "fine" et de référence sont généralement satisfaisants; ils correspondent à ce que l'on pouvait espérer connaissant les différentes données fournies en entrée du processus.

A première vue, la qualité des MNS "fins" peut également sembler suffisante : il est possible de distinguer les différents objets de sol et de sursol. Qui plus est, lorsque l'on calcule la différence entre ces MNS "fins" et le MNS récent de référence, les erreurs systématiques observées en section 4.2.1 sont fortement réduites ( $<2 \mathrm{~m}$ dans les zones n'ayant pas évoluées). Ces écarts entre MNS sont visibles sur la figure 9 pour le jeu de données Fabas 1971. Toutefois, certains problèmes persistent. Ainsi, on continue à observer des discontinuités linéaires présentant des sauts altimétriques. Il s'agit là d'un symptôme du fait que des erreurs d'auto-étalonnage, voire d'estimation des poses des clichés, persistent. Des sauts plus importants existent également près des frontières de la zone d'étude, là où la compensation était moins contrainte. Néanmoins, il est important de garder à l'esprit que ces deux zones d'étude présentent une grande complexité. Par exemple, la mer couvre une partie importante du site de Fréjus. Aussi, il est impossible de détecter des points de liaison et des points d'appui dans ce secteur. Cela a pour conséquence un chantier peu contraint dans cette zone lors du calcul d'aérotriangulation.

\section{Conclusion et perspectives}

Un processus quasiment entièrement automatique a été mis en œuvre pour la remise en géométrie de prises de vues photogrammétriques anciennes. II s'appuie sur différentes informations (très) approchées fournies par les métadonnées associées aux clichés, et s'efforce de les affiner. En particulier, une étape clé de cette chaîne consiste à identifier de manière automatique des points homologues entre ces prises de vues anciennes et une donnée de référence récente, et à les utiliser comme points d'appui pour un calcul d'aérotriangulation.

Cette étape est facilitée par l'utilisation d'ortho-images "grossières" intermédiaires, dont le géoréférencement absolu approché déduit de métadonnées de la prise de vues est suffisant pour réduire la zone de recherche de ces points homologues. En outre, le fait que ces orthoimages anciennes "grossières" soient des ortho-images vraies associées à des MNS permet d'effectuer la recherche de points d'appui simplement entre ortho-images, puis de reprojeter les points détectés dans les clichés anciens afin d'en déduire leurs coordonnées image. Une nouvelle aérotriangulation peut alors être calculée et un nouveau jeu d'ortho-images et MNS "fins" produit. Leur recalage planimétrique par rapport aux données de référence est satisfaisant. L'information altimétrique est également améliorée mais n'est pas encore parfaite.

De nouveaux développements sont envisagés pour améliorer les résultats, en particulier pour détecter des

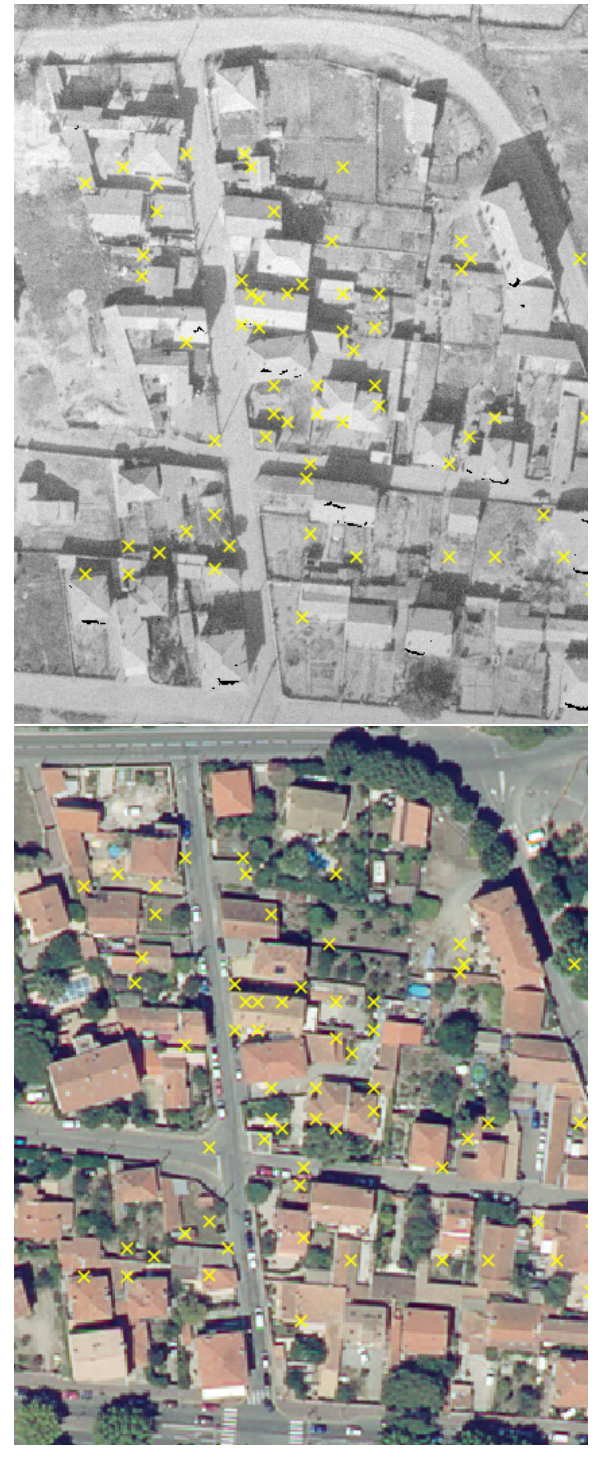

FIGURE 5. Fréjus 1954. Exemples de points d'appui automatiques sur les ortho-images de 1954 (en haut) et de référence (en bas).

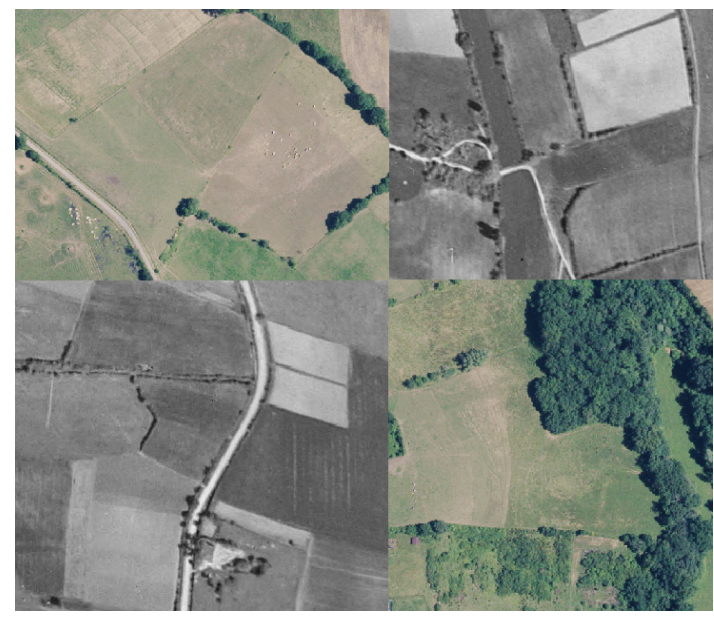

FIgURE 6. Fabas 1942. Damier composé de l'orthoimage de 1942 issue du géoréférencement approché des clichés anciens et de l'ortho-image récente utilisée comme référence. 


\begin{tabular}{|c|c|ccc|ccc|}
\hline Date & nb & $\begin{array}{c}\text { moyenne(x) } \\
(\mathrm{m})\end{array}$ & $\begin{array}{c}\text { moyenne(y) } \\
(\mathrm{m})\end{array}$ & $\begin{array}{c}\text { moyenne(z) } \\
(\mathrm{m})\end{array}$ & $\begin{array}{c}\text { variance(x) } \\
(\mathrm{m})\end{array}$ & $\begin{array}{c}\text { variance(y) } \\
(\mathrm{m})\end{array}$ & $\begin{array}{c}\text { variance(z) } \\
(\mathrm{m})\end{array}$ \\
\hline \multicolumn{7}{|c|}{ Fabas $(12 \mathrm{~km} \times 10 \mathrm{~km})$} \\
\hline 1942 & 3275 & 4,52 & 4,13 & 1,85 & 12,48 & 10,65 & 5,65 \\
1962 & 5688 & 4,21 & 4,04 & 1,86 & 13.07 & 13,08 & 5,34 \\
1971 & 3029 & 2,78 & 2,87 & 1,52 & 6,49 & 6,78 & 2.92 \\
1984 & 3438 & 6,12 & 3,78 & 2,63 & 76,43 & 14,93 & 10,31 \\
1992 & 6222 & 3,15 & 3,46 & 1,99 & 6,79 & 7,07 & 3,60 \\
\hline \multicolumn{7}{|c|}{ Fréjus $(3 \mathrm{~km} \times 2 \mathrm{~km})$} \\
\hline 1954 & 1133 & 1,01 & 0,87 & 1,85 & 0,51 & 0,57 & 3,51 \\
1966 & 5116 & 1,15 & 0,83 & 1,60 & 0,66 & 0,45 & 2,93 \\
1970 & 4661 & 0,86 & 0,93 & 1,84 & 0,94 & 0,86 & 4,30 \\
1978 & 8032 & 2,56 & 1,29 & 2,97 & 4,19 & 1,55 & 6,78 \\
1989 & 18670 & 1,02 & 1,02 & 1,42 & 0,72 & 0,84 & 2,74 \\
\hline
\end{tabular}

TABLE 2. Nombre de points d'appui automatiques détectés (nb) et résidus terrain à l'issue de la mise en place "fine" des clichés par rapport à une référence récente.

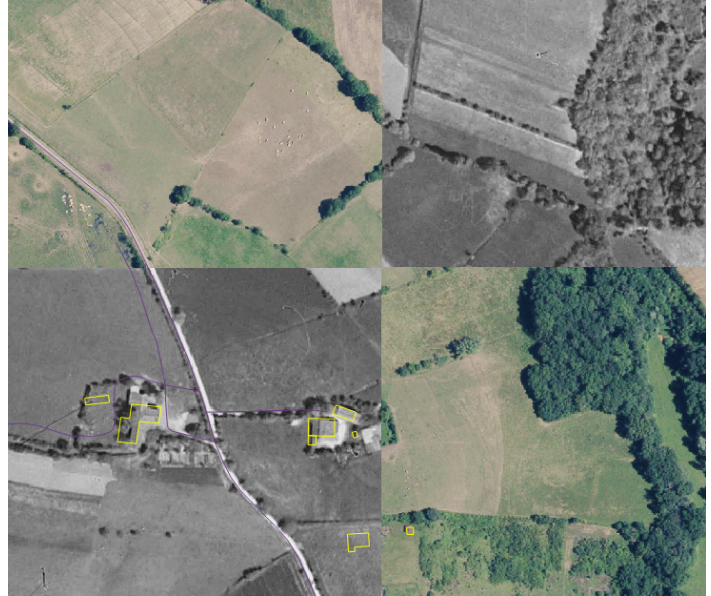

FIGURE 7. Fabas 1942. Damier composé de l'orthoimage de 1942 issue du géoréférencement final des clichés anciens et de l'ortho-image récente utilisée comme référence. Les bâtiments et les routes d'une base de données topographique récente (BDTopo de l'IGN) sont également affichés (en jaune et magenta).

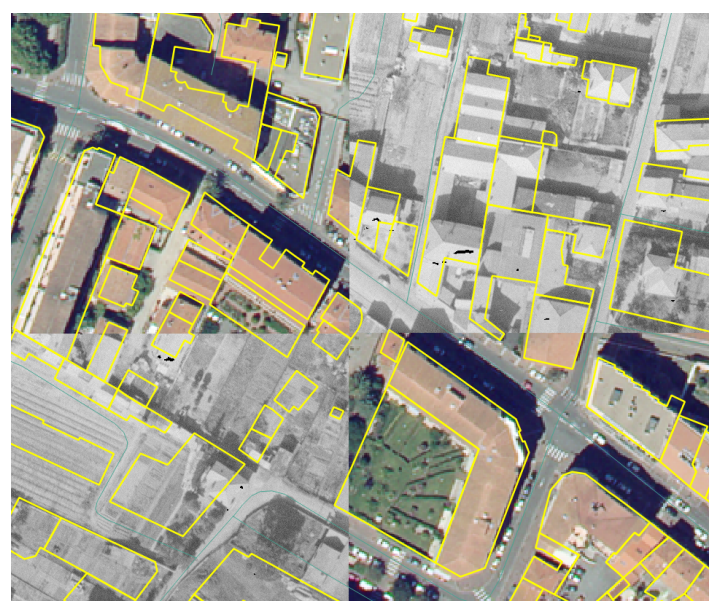

FIGURE 8. Fréjus 1954. Damier composé de l'orthoimage de 1954 issue du géoréférencement final des clichés anciens et de l'ortho-image récente utilisée comme référence. Les bâtiments et les routes d'une base de données topographique récente (BDTopo de l'IGN) sont également affichés (en jaune et magenta).
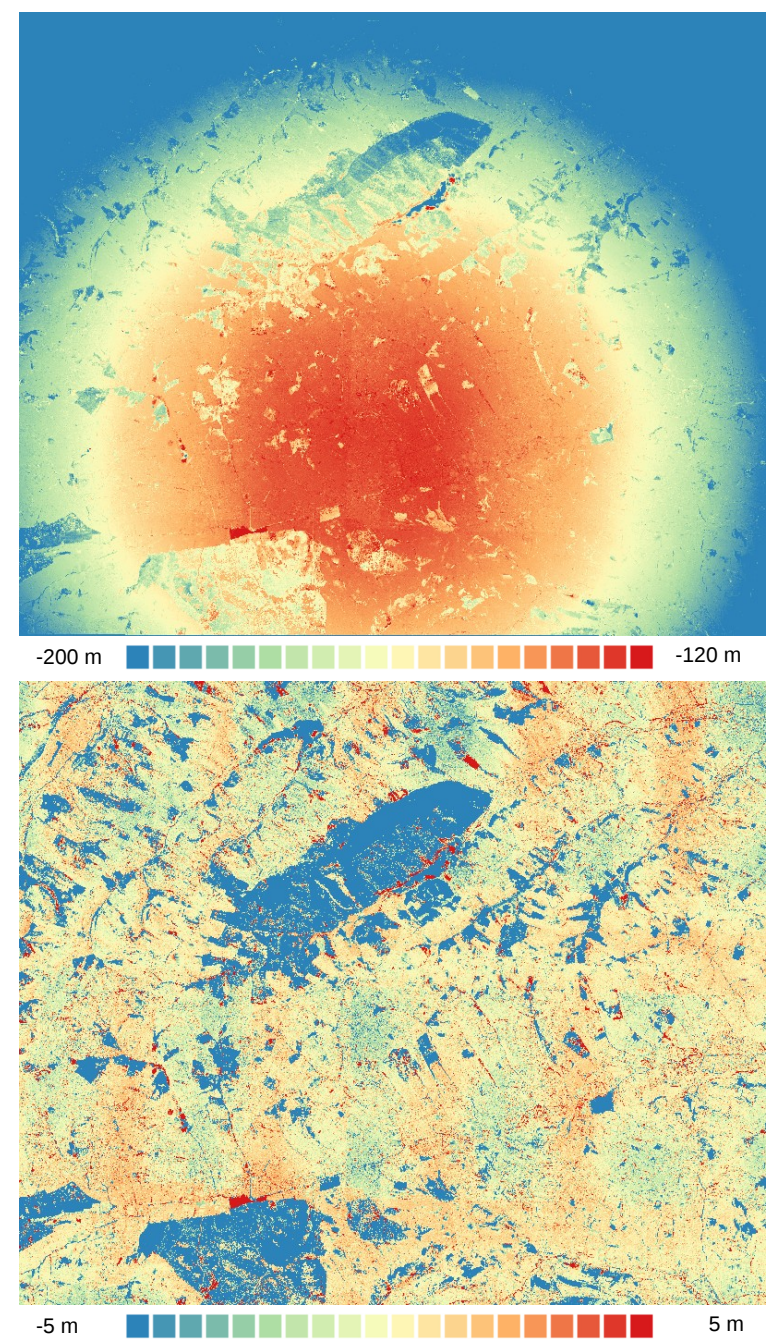

FIGURE 9. Fabas 1971. Différences entre le MNS actuel (référence) et les MNS calculés à partir des clichés de 1971 pour un géoréférencement absolu des images approché (en haut) ou affiné (en bas). 
points d'appui mieux répartis, plus nombreux, plus robustes et meilleurs. Ainsi, davantage de points d'appui pourraient être détectés par d'autres méthodes, comme par exemple celles se fondant sur l'apprentissage profond (Yi et al., 2016). II serait d'ailleurs possible de tirer parti de différents millésimes d'ortho-images déjà géoréférencées pour entraîner une architecture de réseaux de neurones spécifique.

D'autres approches méritent également d'être étudiées. Certains bâtiments constituent des structures pérennes, et donc, une fois le MNS "fin" calculé, il est envisageable de procéder à un recalage 3D entre des patchs issus de ce MNS ancien et du MNS récent de référence. Un tel recalage devrait prendre en compte un score évaluant si un changement 3D s'est produit.

La méthode proposée dans (Cléry et al., 2014) pourrait aussi permettre de mettre en correspondance des segments détectés dans une (ortho-)image ancienne et les objets routes et bâti d'une base de données topographiques. De telles "lignes d'appui" pourraient ensuite constituer de nouvelles observations pour le calcul d'aérotriangulation. Enfin, une fois la précision planimétrique des ortho-images anciennes suffisante pour entraîner des modèles de classification d'occupation du sol à partir de données d'occupation du sol récente, il deviendrait possible de sémantiser grossièrement les données anciennes. Les points d'appui détectés automatiquement se verraient alors attribuer une classe d'occupation du sol (comme par exemple, végétation/non-végétation), et pourraient être filtrées selon cette classe en amont du processus d'aérotriangulation.

\section{Références}

Aguilar, M., Aguilar, F., Fernández, I., Mills, J., 2013. Accuracy assessment of commercial self-calibrating bundle adjustment routines applied to archival aerial photography. The Photogrammetric Record 28 (141), 96-114.

Asner, G., Archer, S., Hughes, R., Ansley, R. J., Wessman, C., 2003. Net changes in regional woody vegetation cover and carbon storage in Texas Drylands, 1937-1999. Global Change Biology 9 (3), 316-335.

Aubry, M., Russell, B. C., Sivic, J., 2014. Painting-to-3D model alignment via discriminative visual elements. ACM Transactions on Graphics 33 (2).

Ayoub, F., Leprince, S., Avouac, J.-P., 2009. Co-registration and correlation of aerial photographs for ground deformation measurements. ISPRS Journal of Photogrammetry and Remote Sensing 64 (6), 551-560.

Cardenal, J., Delgado, J., Mata, E., González, A., Olague, I., 2006. Use of historical flight for landslide monitoring. Dans : Spatial Accuracy, ISARA, 5-7 July, Lisbon, Portugal.

Chen, H.-R., Tseng, Y.-H., et al., 2016. Study of automatic image rectification and registration of scanned historical aerial photographs. The International Archives of the Photogrammetry, Remote Sensing and Spatial Information Sciences 41(B8), 1229-1236.

Cléry, I., Pierrot-Deseilligny, M., Vallet, B., 2014. Automatic georeferencing of a heritage of old analog aerial photographs. ISPRS Annals of Photogrammetry, Remote Sensing and Spatial Information Sciences II-3, 33-40.
Dalal, N., Triggs, B., 2005. Histograms of oriented gradients for human detection. Dans : IEEE Conference on Computer Vision and Pattern Recognition, IEEE, 20-25 June, San Diego, USA.

Ellis, E. C., Wang, H., Xiao, H. S., Peng, K., Liu, X. P., Li, S. C., Ouyang, H., Cheng, X., Yang, L. Z., 2006. Measuring longterm ecological changes in densely populated landscapes using current and historical high resolution imagery. Remote Sensing of Environment 100 (4), 457-473.

Ford, M., 2013. Shoreline changes interpreted from multitemporal aerial photographs and high resolution satellite images : Wotje atoll, marshall islands. Remote Sensing of Environment 135, 130-140.

Fox, A. J., Cziferszky, A., 2008. Unlocking the time capsule of historic aerial photography to measure changes in antarctic peninsula glaciers. The Photogrammetric Record 23 (121), 51-68.

Galster, J. C., Pazzaglia, F. J., Germanoski, D., 2008. Measuring the impact of urbanization on channel widths using historic aerial photographs and modern surveys. Journal of the American Water Resources Association 44 (4), 948-960.

Giordano, S., Le Bris, A., Mallet, C., 2017. Fully automatic analysis of archival aerial images current status and challenges. Dans : Joint Urban Remote Sensing Event (JURSE), IEEE/ISPRS, 6-8 March, Dubai, UAE.

Giordano, S., Le Bris, A., Mallet, C., 2018. Toward automatic georeferencing of archival aerial photogrammetric surveys. ISPRS Annals of Photogrammetry, Remote Sensing and Spatial Information Sciences IV-2, 105-112.

Jao, F.-J., Chu, H.-J., Tseng, Y.-H., 2014. Historical image registration and land-use land-cover change analysis. Environments 1 (2), 181-189.

Kadmon, R., Harari-Kremer, R., 1999. Studying long-term vegetation dynamics using digital processing of historical aerial photographs. Remote Sensing of Environment 68 (2), 164176.

Kim, S., Min, D., Ham, B., Ryu, S., Do, M., Sohn, K., 2015. DASC : Dense Adaptive Self-Correlation descriptor for multimodal and multi-spectral correspondence. Dans : IEEE Conference on Computer Vision and Pattern Recognition, IEEE, 8-10 June, Boston, USA.

Korpela, I., 2006. Geometrically accurate time series of archived aerial images and airborne lidar data in a forest environment. Silva Fennica 40 (1), 109.

Lowe, D., 1999. Object recognition from local scale-invariant features. Dans : Internationl Conference on Computer Vision, IEEE, 20-27 September, Kerkyra, Greece. pp. 1150-1157.

Meyer, P., Staenz, K., Itten, K., 1996. Semi-automated procedures for tree species identification in high spatial resolution data from digitized colour infrared-aerial photography. ISPRS Journal of Photogrammetry and Remote Sensing 51 (1), 516.

Micheletti, N., Lane, S., Chandler, J., 2015. Application of archival aerial photogrammetry to quantify climate forcing of alpine landscapes. The Photogrammetric Record 30 (150), 143-165.

Mondino, E., Chiabrandoa, R., 2008. Multi-temporal block adjustment for aerial image time series : The Belvedere glacier case study. The International Archives of the Photogrammetry, Remote Sensing and Spatial Information Sciences 37 (B2), 89-94.

Nagarajan, S., Schenk, T., 2016. Feature-based registration of historical aerial images by area minimization. ISPRS Journal of Photogrammetry and Remote Sensing 116, 15-23.

Nebiker, S., Lack, N., Deuber, M., 2014. Building change detection from historical aerial photographs using dense image matching and object-based image analysis. Remote Sensing 
6 (9), 8310-8336.

Necsoiu, M., Dinwiddie, C., Walter, G., Larsen, A., Stothoff, S., 2013. Multi-temporal image analysis of historical aerial photographs and recent satellite imagery reveals evolution of water body surface area and polygonal terrain morphology in Kobuk Valley National Park, Alaska. Environmental Research Letters 8 (2), 025007.

Nocerino, E., Menna, F., Remondino, F., 2012. Multi-temporal analysis of landscapes and urban areas. International Archives of the Photogrammetry, Remote Sensing and Spatial Information Sciences 39(B4), 85-90.

Nurminen, K., Litkey, P., Honkavaara, E., Vastaranta, M., Holopainen, M., Lyytikäinen-Saarenmaa, P., Kantola, T., Lyytikäinen, M., 2015. Automation aspects for the georeferencing of photogrammetric aerial image archives in forested scenes. Remote Sensing 7 (2), 1565-1593.

Pierrot Deseilligny, M., Cléry, I., 2011. Apero, an open source bundle adjusment software for automatic calibration and orientation of set of images. International Archives of the Photogrammetry, Remote Sensing and Spatial Information Sciences 38(5/W16), 269-276.

Véga, C., St-Onge, B., 2008. Height growth reconstruction of a boreal forest canopy over a period of 58 years using a combination of photogrammetric and lidar models. Remote Sensing of Environment 112 (4), 1784-1794.

Verdie, Y., Yi, K., Fua, P., Lepetit, V., 2015. TILDE : A temporally invariant learned detector. Dans : IEEE Conference on Computer Vision and Pattern Recognition, IEEE, 8-10 June, Boston, USA. pp. 5279-5288.

Walstra, J., Chandler, J., Dixon, N., Dijkstra, T., 2004. Time for change-quantifying landslide evolution using historical aerial photographs and modern photogrammetric methods. International Archives of Photogrammetry, Remote Sensing and Spatial Information Sciences 35(B4), 475-480.

Yi, K., Trulls, E., Lepetit, V., Fua, P., 2016. LIFT : Learned Invariant Feature Transform. Dans : European Conference on Computer Vision, 8-16 October, Amsterdam, The Netherlands. 